\title{
Clinical Features and Risk Factors of Active Tuberculosis in Patients with Behçet's Disease
}

\author{
Yaxu Liu $\mathbb{D}^{1}{ }^{1}$ Lifan Zhang, ${ }^{1,2,3}$ Ziyue Zhou, ${ }^{1}$ Luxi Sun, ${ }^{4}$ Baotong Zhou, ${ }^{1,3}$ Xiaoqing Liu $\mathbb{D}^{1,2,3}$ \\ and Wenjie Zheng $\mathbb{1}^{4}$ \\ ${ }^{1}$ Department of Infectious Diseases, Peking Union Medical College Hospital, Chinese Academy of Medical Sciences \& Peking Union \\ Medical College, Beijing 100730, China \\ ${ }^{2}$ Peking Union Medical College Hospital, Chinese Academy of Medical Science, Clinical Epidemiology Unit, \\ International Epidemiology Network, Beijing 100730, China \\ ${ }^{3}$ Chinese Academy of Medical Sciences and Peking Union Medical College, Centre for Tuberculosis Research, Beijing 100730, China \\ ${ }^{4}$ Department of Rheumatology, Peking Union Medical College Hospital, Chinese Academy of Medical Sciences \& Peking Union \\ Medical College, The Ministry of Education Key Laboratory, National Clinical Research Center for Dermatologic and \\ Immunologic Diseases, Beijing 100730, China
}

Correspondence should be addressed to Xiaoqing Liu; liuxqpumch@126.com and Wenjie Zheng; wenjzheng@gmail.com

Received 29 July 2020; Revised 20 October 2020; Accepted 27 October 2020; Published 24 November 2020

Academic Editor: Roberta Antonia Diotti

Copyright ( 2020 Yaxu Liu et al. This is an open access article distributed under the Creative Commons Attribution License, which permits unrestricted use, distribution, and reproduction in any medium, provided the original work is properly cited.

\begin{abstract}
To investigate the clinical features and potential risk factors of active tuberculosis (ATB) in Behçet's disease (BD), we conducted a case-control study on hospitalized BD patients in our institute from 2010 to 2019. BD patients with ATB were enrolled as the case group. The control group was selected by random number sampling from the remaining BD patients, including those with latent tuberculosis infection, previous tuberculosis, or without tuberculosis. Finally, we reviewed 386 BD patients and identified 21 (5.4\%) ATB cases, including four (19.0\%) microbiologically confirmed and 17 (81.0\%) clinically diagnosed. We found that BD patients with ATB were more prone to have systemic symptoms (fever, night sweating, and unexplained weight loss) and/or symptoms related to the infection site. Multivariate logistic regression analysis revealed that erythrocyte sedimentation rate $($ ESR $)>60$ $\mathrm{mm} / \mathrm{h}(\mathrm{OR}=13.710,95 \% \mathrm{CI}(1.101,170.702))$, increased $\mathrm{IgG}(\mathrm{OR}=1.226,95 \% \mathrm{CI}(1.001,1.502))$, and positive T-SPOT.TB $\left(\mathrm{OR}=7.793,95 \% \mathrm{CI}(1.312,48.464)\right.$, for $24-200 \mathrm{SFC} / 10^{6} \mathrm{PBMC}$; OR $=17.70595 \% \mathrm{CI}(2.503,125.260)$, for $\left.>200 \mathrm{SFC} / 10^{6} \mathrm{PBMC}\right)$ were potential risk factors for ATB in BD patients. Our study suggested that when BD patients have systemic symptoms with significantly elevated TB-SPOT, the diagnosis of ATB should be considered.
\end{abstract}

\section{Introduction}

Behçet's disease (BD) is a systemic vasculitis prevalent in the areas along the Ancient Silk Road such as Turkey, Iran, China, and Japan [1]. In China, the prevalence of BD is estimated to be 14 in 100000 [2]. It has been noticed that $\mathrm{BD}$ is closely related to tuberculosis (TB) $[3,4]$, which is also prevalent in China [5].

$\mathrm{TB}$ infection may trigger the pathogenesis of $\mathrm{BD}[6,7]$, and $\mathrm{BD}$ patients are at high risk of active tuberculosis (ATB) infection $[8,9]$. The treatment of $\mathrm{BD}$ generally includes glucocorticoid, immunosuppressant, and biologic agents [10-12].
Once a BD patient is infected with ATB, the immunosuppressive therapy would be reduced or suspended [13], resulting in the BD deterioration and increased risk of developing immune reconstitution inflammatory syndrome [14]. If the ATB is not promptly diagnosed or properly treated, the intensive immunosuppressive therapy may lead to disseminated TB and severe consequences [15]. Thus, understanding the clinical features and risk factors of ATB in patients with $\mathrm{BD}$ is of great importance.

To date, related studies are very limited. Lin et al. reported the clinical features of ATB in $10 \mathrm{BD}$ patients, but the BD sample size for the study was only 37 , and the 
statistical analysis was not conducted [16]. Liu compared the clinical features of $36 \mathrm{BD}$ patients with ATB and $407 \mathrm{BD}$ patients without TB. Liu discovered that ATB patients were more prone to fever, arthritis, thrombosis, and elevated IgG [17]. However, patients with latent tuberculosis infection (LTBI) or previous tuberculosis (PTB) were not included in the study. Moreover, to the best of our knowledge, there is no study investigating the risk factor of ATB in BD patients. Therefore, a case-control study was conducted to investigate the clinical features and explore the risk factors of ATB in BD patients.

\section{Materials and Methods}

2.1. Participants. Medical records of all hospitalized patients diagnosed with BD in Peking Union Medical College Hospital (PUMCH) from January 1, 2010, to June 30, 2019, were retrospectively reviewed. All patients fulfilled the 2013 International Criteria for Behçet's Disease (ICBD) [18]. Patients suspected of tuberculosis infection underwent careful examinations, including pathological examinations (culture and acid-fast staining of sputum and other available samples like pleural effusion), a biopsy of the suspected organs (colonoscopy of the colorectal lesions, the surgical sample of the vertebra), and immunological examinations (T-SPOT.TB test), for the evidence of tuberculosis infection as well as the exclusion of other common infections in BD like bacteria and CMV. The categorization of TB was based on the 2000 criteria [19] and those applied in previous studies [20, 21] (see Table S1 in the Supplementary Material online). Two experienced experts of infectious diseases were asked to review the cases and check the diagnosis of ATB independently. Only confirmed by both experts would the clinical diagnosis of ATB be accepted. All patients diagnosed with $\mathrm{BD}$ and ATB were enrolled as the case group. The remaining patients with $\mathrm{BD}$ were ranked according to the date of admission, and the random samples were chosen using random number tables as the control group.

2.2. Data Collection. The demographic features (age and sex), past medical history (the course and treatment of BD before hospitalization, previous infection of TB, etc.), current treatment (glucocorticoid dosage, immunosuppressant, etc.), BD systemic involvement, TB-related systemic symptoms (fever, cough, etc.), laboratory results (complete blood cell count, TSPOT.TB, etc.), and comorbidities of the patients were collected and analyzed. The flowchart of the study was shown in Figure 1.

2.3. Ethic Review. This study complied with the Declaration of Helsinki and was approved by the Ethics Committee of PUMCH (ethics approval number: S-715). Informed consent was obtained from all patients for being included in the study.

2.4. Statistical Analyses. Continuous variables were examined by the Kolmogorov-Smirnov test, and those with normal distribution were expressed as mean \pm standard deviation (SD), while those without were described as median and interquartile range (IQR). Categorical variables were pre- sented as numbers and percentages. Comparisons of continuous variables were performed using Student's $t$-test when they were in normal distribution, and Mann-Whitney $U$ -test when they were not. Categorical data were compared using the Chi-squared test or Fisher's exact test. $p<0.05$ was considered to be statistically significant.

Variables with $p<0.1$ in the univariate analysis were considered candidate risk factors, and those with clinical significance were evaluated with stepwise binary logistic regression analysis (inclusion threshold $p<0.05$, exclusion threshold $p>0.1$ ). The odds ratio (OR) of the risk factors and its 95\% confidential interval (CI) were calculated. All statistical analyses were performed with SPSS 16.0 (SPSS Inc., USA).

\section{Results}

3.1. General Data. Three hundred eighty-six hospitalized patients were diagnosed with BD in PUMCH from January 2010 to June 2019. Twenty-one (5.4\%) of them were confirmed with the diagnosis of ATB, including four microbiologically confirmed and 17 clinically diagnosed. The control group consisted of 69 patients, including 25 (36.2\%) with LTBI or PTB and 44 (63.8\%) without TB.

3.2. Clinical Features of BD with ATB. Four of the 21 ATB patients were microbiologically confirmed while 17 were clinically diagnosed (see Table S2 in the Supplementary Material online). Sixteen (76.2\%) patients had pulmonary TB, four (19.0\%) had extrapulmonary TB, and one patient's infection site could not be identified. Four patients $(19.0 \%)$ had more than one organ involved. The involved organs other than the lung included the gastrointestinal tract (2, $9.5 \%)$, bones $(2,9.5 \%)$, lymph nodes $(1,4.8 \%)$, larynx (1, $4.8 \%)$, peritoneum $(1,4.8 \%)$, and pericardium $(1,4.8 \%)$.

Patients with ATB were more likely to develop systematic symptoms including fever $(18,85.7 \%)$, night sweating (8, $38.1 \%)$, and unexplained weight loss (13, 61.9\%). Meanwhile, many ATB patients also had symptoms related to the site of infection. For example, pulmonary ATB patients had a productive cough; osteal ATB patients had bone pain and restricted range of motion; lymphatic ATB patients had lymphadenopathy and sinus formation; pharyngeal ATB patients had mucosal erosions, ulcers, and tubercles; and gastrointestinal ATB patients had abdominal pain, diarrhea, and/or constipation.

All ATB patients showed abnormalities in chest radiology, including nodules, cord, or patches, predominantly in the upper and medial lobes. Some other abnormalities can also be revealed by CT scan, such as lymph node enlargement with a low-density core in lymphatic ATB, vertebral damage in osteal ATB, and swelling of gastrointestinal walls in gastrointestinal ATB. The pericardial ATB patients also showed fibrinous pericardial effusion on echocardiogram.

All BD patients with ATB had clinical improvement after anti-TB treatment. Moreover, with intensive treatment of $\mathrm{BD}$ following the control of ATB, patients had achieved long-term stability of their disease condition during the follow-up period. 


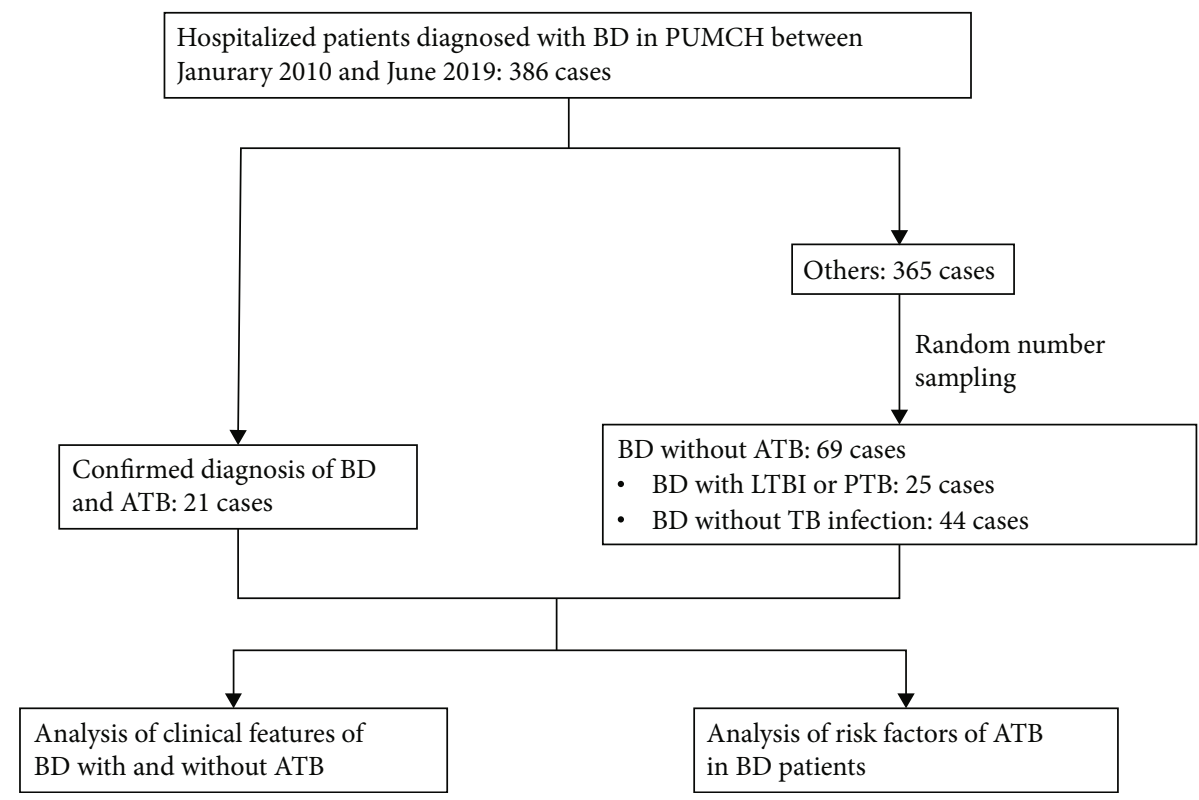

FIgure 1: Flowchart of the study. BD: Behçet's disease; PUMCH: Peking Union Medical College Hospital; ATB: active tuberculosis; LTBI: latent tuberculosis infection; PTB: previous tuberculosis.

\subsection{Clinical Comparison of BD Patients with and without $A T B$}

3.3.1. Past Medical History and Medication. More ATB patients had evidence of previous TB infection (13 $(61.90 \%)$ vs. $17(24.64 \%), p=0.002)$. The duration of previous glucocorticoid use was shorter in the ATB group $(0(0,3.5)$ month vs. $3(0,13)$ months, $p=0.028)$ (Table 1$)$. The case and control groups showed no difference in immunosuppressant or biologics treatment (Table 1) and comorbidities (diabetes mellitus: $4.76 \%$ vs. $7.25 \%, p=1.00$; myelodysplastic syndrome: $4.76 \%$ vs. $7.25 \%, p=1.00$; and others: $52.38 \%$ vs. $52.17 \%, p=1.00)$.

3.3.2. Symptoms and Signs. The systemic involvement of BD patients with and without ATB revealed no difference (Table 2), but more ATB patients had TB toxic symptoms like fever $(85.7 \%$ vs. $49.3 \%, p=0.003)$, cough $(38.1 \%$ vs. $7.2 \%, p=0.002)$, expectoration $(33.3 \%$ vs. $4.3 \%, p=0.001)$, and night sweating $(38.1 \%$ vs. $5.8 \%, p=0.001)$.

3.3.3. Laboratory Tests. ATB patients had increased level of erythrocyte sedimentation rate (ESR, $31(22,57)$ vs. $16(6$, 39) $\mathrm{mm} / \mathrm{h}, p=0.004)$, hypersensitive $\mathrm{C}$ reactive protein (hsCRP, $28.32(8.50,63.83)$ vs $10.37(1.61,43.59) \mathrm{mg} / \mathrm{L}, p=$ $0.038)$, immunoglobulin $\mathrm{G}$ (IgG, 12.55 (9.98, 15.61) vs. 9.6 $(7.84,13.13) \mathrm{g} / \mathrm{L}, p=0.006)$, complement 3 (C3, $1.3048 \pm$ 0.23713 vs. $1.1664 \pm 0.24768 \mathrm{~g} / \mathrm{L}, p=0.036)$, and complement 4 (C4, $0.2644 \pm 0.09852$ vs. $0.2213 \pm 0.03997 \mathrm{~g} / \mathrm{L}, p=$ $0.039)$, higher positive rate of T-SPOT.TB (17 (80.95\%) vs. $19(27.54 \%), p=0.000)$, and elevated SFC in T-SPOT.TB test (336 $(92,1084) \mathrm{SFC} / 10^{6} \mathrm{PBMC}$ vs. $0(0,27) \mathrm{SFC} / 10^{6} \mathrm{PBMC}$, $p=0.000$ ) (Table 2). Complete blood cell count and the liver and kidney function tests revealed no significant difference.
3.3.4. Risk Factors for ATB in BD Patients. The result of logistic regression analysis is shown in Table 3 . ESR $>60 \mathrm{~mm} / \mathrm{h}$ $(\mathrm{OR}=13.710,95 \% \mathrm{CI}(1.101,170.702), p=0.042)$, increased $\operatorname{IgG}(\mathrm{OR}=1.226,95 \% \mathrm{CI}(1.001,1.502), p=0.049)$, and positive T-SPOT.TB (OR $=7.793,95 \%$ CI $(1.312,48.464), p=$ 0.024 , for $24-200 \mathrm{SFC} / 10^{6} \mathrm{PBMC} ; \mathrm{OR}=17.705$ (2.503, $125.260), p=0.004$, for $\left.>200 \mathrm{SFC} / 10^{6} \mathrm{PBMC}\right)$ were found to be statistically significant.

\section{Discussion}

This study is the first well-designed case-control study investigating the clinical features of BD patients with ATB and exploring the potential risk factors of ATB in BD patients. We demonstrated that $\mathrm{BD}$ patients tend to develop pulmonary ATB and have multiple sites involved. Besides presenting with the symptoms related to the infection sites, ATB patients also had systemic symptoms, including fever, night sweating, and unexplained weight loss. Furthermore, logistic regression analysis indicates that ESR $>60 \mathrm{~mm} / \mathrm{h}$, increased IgG, and positive T-SPOT.TB are potential risk factors of ATB in BD patients.

Even with thorough examinations, most ATB patients in our study were clinically diagnosed and lacked the microbiological evidence, which is attributed to the high prevalence of TB in China and the complicated nature of the cases referred to our hospital. Microbiological examinations, including bacterial culture, acid-fast staining, and molecular tests, are currently the gold standard for ATB diagnosis. However, the sensitivity of these tests is not satisfactory [22]. In countries with heavy tuberculosis burden, culture- or smear-negative cases are not rare, sometimes even accounting for more than $70 \%$ of all ATB cases $[20,23,24]$. Other tests like acute inflammatory markers are not specific, while T-SPOT.TB cannot differentiate ATB from LTBI/PTB [25]. Thus, all 
TABLe 1: Past medical history and medication of BD patients with and without ATB.

\begin{tabular}{|c|c|c|c|}
\hline & BD with ATB $(n=21)$ & BD without ATB $(n=69)$ & $p$ \\
\hline Sex (male, \%) & $13(61.90 \%)$ & $35(50.72 \%)$ & 0.369 \\
\hline Age $(M \pm \mathrm{SD})$ & $36.19 \pm 12.46$ & $38.58 \pm 12.68$ & 0.450 \\
\hline \multicolumn{4}{|l|}{ Past medical history } \\
\hline Previous contact with ATB patients (\%) & $3(14.29 \%)$ & $2(2.90 \%)$ & 0.081 \\
\hline Previous prophylactic treatment of TB (\%) & $1(4.76 \%)$ & $3(4.35 \%)$ & 1.000 \\
\hline Evidence of $\mathrm{PTB}^{\mathrm{a}}(\%)$ & $13(61.90 \%)$ & $17(24.64 \%)$ & 0.002 \\
\hline \multicolumn{4}{|l|}{ Previous treatment of $\mathrm{BD}$} \\
\hline \multicolumn{4}{|l|}{ Glucocorticoid } \\
\hline Maximal dosage (mg/d, median, IQR $)^{\mathrm{b}}$ & $0(0,50)$ & $25(0,60)$ & 0.093 \\
\hline Duration (months, median, IQR) & $0(0,3.5)$ & $3(0,13)$ & 0.028 \\
\hline \multicolumn{4}{|l|}{ Biologics } \\
\hline Infliximab (\%) & $2(9.52 \%)$ & $2(2.90 \%)$ & 0.231 \\
\hline Other TNF- $\alpha$ inhibitors (\%) & $2(9.52 \%)$ & $3(4.35 \%)$ & 0.587 \\
\hline \multicolumn{4}{|l|}{ Immunosuppressant } \\
\hline CTX (\%) & $5(23.81 \%)$ & $11(15.94 \%)$ & 0.515 \\
\hline CsA (\%) & $0(0 \%)$ & $10(14.49 \%)$ & 0.109 \\
\hline MTX (\%) & $1(4.76 \%)$ & $3(4.35 \%)$ & 1.000 \\
\hline FK506 (\%) & $1(4.76 \%)$ & $2(2.90 \%)$ & 0.554 \\
\hline AZA (\%) & $2(9.52 \%)$ & $5(7.25 \%)$ & 0.663 \\
\hline \multicolumn{4}{|l|}{ Current treatment of $\mathrm{BD}^{\mathrm{c}}$} \\
\hline Glucocorticoid dosage (mg/d, median, IQR) ${ }^{\mathrm{b}}$ & $0(0,15)$ & $5(0,32.5)$ & 0.177 \\
\hline \multicolumn{4}{|l|}{ Biologics } \\
\hline Infliximab (\%) & $0(0 \%)$ & $2(2.90 \%)$ & 1.000 \\
\hline Other TNF- $\alpha$ inhibitor (\%) & $1(4.76 \%)$ & $0(0 \%)$ & 0.233 \\
\hline \multicolumn{4}{|l|}{ Immunosuppressant } \\
\hline CTX (\%) & $1(4.76 \%)$ & $11(15.94 \%)$ & 0.281 \\
\hline CsA (\%) & $1(4.76 \%)$ & $7(10.14 \%)$ & 0.675 \\
\hline MTX (\%) & $0(0 \%)$ & $0(0 \%)$ & - \\
\hline FK506 (\%) & $1(4.76 \%)$ & $1(1.45 \%)$ & 0.414 \\
\hline AZA (\%) & $1(4.76 \%)$ & $1(1.45 \%)$ & 0.414 \\
\hline
\end{tabular}

${ }^{\mathrm{a}}$ Evidence of PTB includes past history of TB infection and radiological features indicating PTB in chest CT. ${ }^{\mathrm{b}}$ All forms of glucocorticoid were converted to the equivalent dosage of prednisone. ${ }^{\mathrm{c} C u r r e n t}$ use of immunosuppressant and biologics indicates continuous use of the drug for 3 months before hospitalization, and the dosage of glucocorticoid records the maximal dose of glucocorticoid within 2 weeks before hospitalization. TNF- $\alpha$ : tumor necrosis factor- $\alpha$; CTX: cyclophosphamide; CsA: ciclosporin A; MTX: methotrexate; FK506: tacrolimus; AZA: azathioprine; LEF: leflunomide; MMF: mycophenolate mofetilhs.

patients who were highly suspected of ATB but had negative results in microbiological examinations would receive diagnostic anti-TB treatment for 3 months, and their responses to the treatment were documented to help the clinical diagnosis of ATB. Only by being confirmed by two infectious disease experts would the clinical diagnosis of ATB be accepted. In this way, we believe the clinical diagnosis of ATB in our study is reliable. Besides, our study enrolled BD patients with LTBI, PTB, and non-TB as the control group. Compared with previous case reports of patients mostly culture confirmed $[26,27]$ and the case-control studies lacking LTBI/PTB patients in the control group [17], our study population is closer to the actual clinical situation.

Generally, patients with ATB are more likely to experience the symptoms related to the site of infection. Therefore, $\mathrm{TB}$ infection should be suspected when a BD patient pre- sented with the manifestations rarely occurring in $\mathrm{BD}$, such as productive cough, bone pain, restricted range of motion, and lymphadenopathy. Severe systemic symptoms (fever, night sweating, and unexplained weight loss) are also important clues of ATB. It is challenging to identify active TB in BD patients when they presented with manifestations shared by BD and ATB, such as erythema nodosa, pericarditis, and gastrointestinal ulcers predominant at the ileocecal junction. Laboratory tests can be helpful in this situation. Patients with markedly elevated ESR, hsCRP, IgG, and/or positive TSPOT.TB should be considered to have ATB, which is consistent with previous case reports [28] and studies [17,29]. The involvement of lungs in BD generally presents as pulmonary artery thrombosis or aneurysm $[30,31]$, which could be easily differentiated from tuberculosis by chest CT, while the pulmonary parenchymal involvement in $\mathrm{BD}$ is rare. Empiric 
TABLE 2: Clinical presentation and laboratory results of BD patients with and without ATB.

\begin{tabular}{lccr}
\hline & BD with ATB $(n=21)$ & BD without ATB $(n=69)$ & $p$ \\
\hline Systemic involvement of BD & & & \\
Oral ulceration (\%) & $19(90.5 \%)$ & $68(98.6 \%)$ & 0.135 \\
Genital ulceration (\%) & $18(85.7 \%)$ & $49(71.0 \%)$ & 0.176 \\
Erythema nodosa (\%) & $12(57.1 \%)$ & $30(43.5 \%)$ & 0.272 \\
Ocular lesions (\%) & $4(19.0 \%)$ & $25(36.2 \%)$ & 0.140 \\
Vascular manifestations (\%) & $4(19.0 \%)$ & $25(36.2 \%)$ & 0.140 \\
Gastrointestinal involvement (\%) & $6(28.6 \%)$ & $24(34.8 \%)$ & 0.597 \\
CNS involvement (\%) & $3(14.3 \%)$ & $15(21.7 \%)$ & 0.548 \\
Symptoms related to TB infection & & & \\
Fever (\%) & $18(85.7 \%)$ & $34(49.3 \%)$ & 0.003 \\
Cough (\%) & $8(38.1 \%)$ & $5(7.2 \%)$ & 0.002 \\
Expectoration (\%) & $7(33.3 \%)$ & $3(4.3 \%)$ & 0.001 \\
Night sweating (\%) & $8(38.1 \%)$ & $4(5.8 \%)$ & 0.001 \\
Weight loss (\%) & $13(61.9 \%)$ & $28(40.6 \%)$ & 0.086 \\
Laboratory tests & & & \\
ESR (mm/h, median, IQR) & $31(22,57)$ & $16(6,39)$ & 0.004 \\
hsCRP (mg/L, median, IQR) & $28.32(8.50,63.83)$ & $10.37(1.61,43.59)$ & 0.038 \\
IgG (g/L, median, IQR) & $12.55(9.98,15.61)$ & $9.6(7.84,13.13)$ & 0.006 \\
IgA (g/L, median, IQR) & $2.78(1.75,3.66)$ & $2.28(1.59,3.04)$ & 0.286 \\
IgM (g/L, median, IQR) & $0.91(0.71,1.74)$ & $0.93(0.71,1.33)$ & 0.575 \\
Positive T-SPOT.TB (\%) & $17(80.95 \%)(n=19)$ & $19(27.54 \%)(n=66)$ & 0.000 \\
T-SPOT.TB value (SFC/10 6 PBMC, median, IQR) & $336(92,1084)$ & $0(0,27)$ & 0.000 \\
\hline ESR & & \\
\hline
\end{tabular}

ESR: erythrocyte sedimentation rate; hsCRP: hypersensitive C reactive protein; IgG: immunoglobulin G; IgA: immunoglobulin A; IgM: immunoglobulin M; SFC: spot-forming cells; PBMC: peripheral blood mononuclear cells.

TABLE 3: Potential risk factors for ATB in BD patients.

\begin{tabular}{|c|c|c|c|c|c|}
\hline & $b$ & $\mathrm{SE}(b)$ & Wald & $p$ & OR $(95 \% \mathrm{CI})$ \\
\hline \multicolumn{6}{|c|}{$\operatorname{ESR}(\mathrm{mm} / \mathrm{h})$} \\
\hline $0-20$ & & & 4.658 & 0.097 & \\
\hline $20-60$ & 1.719 & 0.965 & 3.178 & 0.075 & $5.581(0.843,36.960)$ \\
\hline$>60$ & 2.618 & 1.287 & 4.141 & 0.042 & $13.710(1.101,170.702)$ \\
\hline $\operatorname{IgG}(\mathrm{g} / \mathrm{L})$ & 0.204 & 0.104 & 3.876 & 0.049 & $1.226(1.001,1.502)$ \\
\hline \multicolumn{6}{|c|}{ T-SPOT.TB (SFC/10 $\left.{ }^{6} \mathrm{PBMC}\right)$} \\
\hline$<24$ & & & 9.266 & 0.010 & \\
\hline $24-200$ & 2.076 & 0.921 & 5.084 & 0.024 & $7.793(1.312,48.464)$ \\
\hline$>200$ & 2.874 & 0.998 & 8.288 & 0.004 & $17.705(2.503,125.260)$ \\
\hline
\end{tabular}

OR: odds ratio; CI: confidential interval.

anti-TB treatment can be considered for patients with the abovementioned clues of ATB. Meanwhile, the response to anti-TB therapy could help the clinical diagnosis or exclusion of ATB.

ESR $>60 \mathrm{~mm} / \mathrm{h}$, increased IgG, and positive T-SPOT.TB were found to be potential risk factors for ATB in patients with BD by logistic regression analysis. ESR was reported to be markedly [27] or slightly [32] increased in ATB patients, and increased IgG in ATB patients had also been reported in Wang's case report [28] and Liu's case-control study [17]. However, prospective cohort studies are needed to understand whether the increase of these nonspecific inflam- matory markers is the cause or the result of ATB. T-SPOT.TB is an interferon-gamma release assay (IGRA) based on the MTB-specific $T$ cell response [25]. The logistic analysis revealed a higher OR value as spot forming cells (SFC) of T-SPOT.TB increases. Even though T-SPOT.TB could not differentiate LTBI and ATB [25], many studies had shown more robust $\mathrm{T}$ cell immune response in ATB patients [20, 33], supporting that positive or markedly increased $\mathrm{T}$ SPOT.TB can be a potential risk factor of ATB in patients with BD.

Notably, our study revealed a smaller dosage and shorter glucocorticoid treatment duration in ATB patients, which is different from previous studies [34]. This may be due to the retrospective design of our study, when patients not excluded for ATB may have avoided using glucocorticoids during the previous treatment. TNF- $\alpha$ inhibitor use, though identified as a risk factor of ATB in previous studies [9], revealed no significant difference between cases and controls in our study. On the one hand, this might be related to the limited sample number in this study; on the other hand, this could be attributed to the thorough screening and proper prophylaxis of patients using TNF- $\alpha$ inhibitors, according to the guidelines $[11,13,35,36]$ published in the 2000s.

We acknowledge some limitations in our study. First, our sample size was relatively small, and most ATB cases were diagnosed according to clinical criteria rather than culture confirmed, which might introduce a risk of bias. Second, 
our center is a national referral center for complicated and critical cases, which might induce a potential selection bias, and the extension of the conclusion must be drawn carefully. Third, the risk factors identified in this case-control study are still required to be confirmed by prospective cohort studies in the future. Finally, most $(81.0 \%)$ of the ATB cases in this study were clinically diagnosed without microbiological evidence. Even though the clinical diagnosis was carefully reviewed and seemed to be plausible in a country with high prevalence of tuberculosis, the diagnosis without a gold standard might introduce a high risk of bias.

\section{Conclusion}

The diagnosis of ATB should be considered when BD patients presented with systemic symptoms like fever, night sweating, and unexplained weight loss, as well as rare presentations of $\mathrm{BD}$ such as productive cough, lymphadenopathy, and bone pain. Significantly elevated ESR, hsCRP, IgG, and positive T-SPOT.TB supports the diagnosis of ATB. Furthermore, the markedly increased SFC in T-SPOT.TB indicates a high risk factor of $\mathrm{ATB}$ in $\mathrm{BD}$ patients.

\section{Data Availability}

The clinical data used to support the findings of this study are included in the article.

\section{Conflicts of Interest}

The authors declare that there is no conflict of interest regarding the publication of this paper.

\section{Acknowledgments}

This work was supported by the National Science and Technology Major Project of the People's Republic of China (grant number 2017ZX10201302), CAMS Initiative for Innovative Medicine (grant number 2016-I2M-1-013), the CAMS Innovation Fund for Medical Sciences (grant number 2019I2M-2-005), National Natural Science Foundation of China (grant number 81871299), National Key Research and Development Program: 'Precise Medical Research' (grant number 2016YFC0906201).

\section{Supplementary Materials}

The supplementary materials include two tables. Table S1: The diagnostic criteria for different TB infection statuses. Table S2: the detailed information of $21 \mathrm{BD}$ patients diagnosed with ATB. (Supplementary materials)

\section{References}

[1] F. Davatchi, C. Chams-Davatchi, H. Shams et al., "Behcet's disease: epidemiology, clinical manifestations, and diagnosis," Expert Review of Clinical Immunology, vol. 13, no. 1, pp. 5765, 2016.

[2] J. N. Kim, S. G. Kwak, J. Y. Choe, and S. K. Kim, "The prevalence of Behçet's disease in Korea: data from Health Insurance
Review and Assessment Service from 2011 to 2015," Clinical and Experimental Rheumatology, vol. 108, no. 6, pp. 38-42, 2017.

[3] P. R. Botha and C. F. N. Koegelenberg, "Risk Factors for Tuberculosis in Patients Receiving Tumour Necrosis Factor$\alpha$ Blockers: What Do We Really Know?," Respiration, vol. 90, no. 3, pp. 185-186, 2015.

[4] Y. Zhou and Z. Lv, "The relationship between Behcet disease and tuberculosis infection," Clin Focus., vol. 24, no. 19, pp. 1742-1744, 2009.

[5] WHO tuberculosis report, 2019, 2019 [Available from: https:// www.who.int/tb/publications/global_report/en/.

[6] H. Direskeneli, "Innate and Adaptive Responses to Heat Shock Proteins in Behcet's Disease," Genetics Research International, vol. 2013, Article ID 249157, 6 pages, 2013.

[7] L. Zhang, Y. Xu, Y. Peng, B. Yan, and Y. Liu, "Behçet's diseaselike syndrome secondary to microbial infection: a case report and review of the literature," International Journal of Clinical and Experimental Pathology, vol. 8, no. 10, pp. 13619-13624, 2015.

[8] S. Borekci, E. Atahan, D. Demir Yilmaz et al., "Factors affecting the tuberculosis risk in patients receiving anti-tumor necrosis Factor- $\alpha$ treatment," Respiration, vol. 90, no. 3, pp. 191-198, 2015.

[9] B. Kisacik, O. N. Pamuk, A. M. Onat et al., "Characteristics predicting tuberculosis risk under tumor necrosis factor- $\alpha$ inhibitors: report from a large multicenter cohort with high background prevalence," The Journal of Rheumatology, vol. 43, no. 3, pp. 524-529, 2016.

[10] T. Hisamatsu, F. Ueno, T. Matsumoto et al., "The 2nd edition of consensus statements for the diagnosis and management of intestinal Behçet's disease: indication of anti-TNF $\alpha$ monoclonal antibodies," Journal of Gastroenterology, vol. 49, no. 1, pp. 156-162, 2014.

[11] G. Hatemi, A. Silman, D. Bang et al., "EULAR recommendations for the management of Behcet disease," Annals of the Rheumatic Diseases, vol. 67, no. 12, pp. 1656-1662, 2008.

[12] G. Hatemi, R. Christensen, D. Bang et al., "2018 update of the EULAR recommendations for the management of Behçet's syndrome," Annals of the Rheumatic Diseases, vol. 77, no. 6, pp. 808-818, 2018.

[13] British Thoracic Society Standards of Care Committee, "BTS recommendations for assessing risk and for managing Mycobacterium tuberculosis infection and disease in patients due to start anti-TNF- treatment," Thorax, vol. 60, no. 10, pp. 800-805, 2005.

[14] C. C. Dobler, "Biologic Agents and Tuberculosis," Microbiology Spectrum, vol. 4, no. 6, 2016.

[15] Y. Kobashi, M. Fukuda, K. Yoshida, and M. Oka, “An indeterminate QuantiFERON TB-2G response for miliary tuberculosis, due to severe pancytopenia," Journal of Infection and Chemotherapy, vol. 13, no. 6, pp. 414-417, 2007.

[16] Y. Lin, G. Yin, and Y. Shu, "Analysis of 10 cases of Behcet disease with tuberculosis infection," West Chin Med J, vol. 23, no. 1, pp. 125-126, 2008.

[17] L. Yuanyuan, "Analysis of the clinical features of Behcet disease related to tuberculosis infection," J Clin Inter Med, vol. 9, no. 35, pp. 615-617, 2018.

[18] International Team for the Revision of the International Criteria for Behcet's Disease, "The International Criteria for Behcet's Disease (ICBD): a collaborative study of 27 countries on 
the sensitivity and specificity of the new criteria," J Eur Acad Dermatol Venereol, vol. 28, no. 3, pp. 338-347, 2014.

[19] "Diagnostic Standards and Classification of Tuberculosis in Adults and Children. This official statement of the American Thoracic Society and the Centers for Disease Control and Prevention was adopted by the ATS Board of Directors, July 1999. This statement was endorsed by the Council of the Infectious Disease Society of America, September 1999," American Journal of Respiratory and Critical Care Medicine, vol. 161, no. 4, pp. 1376-1395, 2000.

[20] X. Wu, P. Chen, W. Wei et al., "Diagnostic value of the interferon- $\gamma$ release assay for tuberculosis infection in patients with Behçet's disease," BMC Infectious Diseases, vol. 19, no. 1, p. 323, 2019.

[21] H. S. Whitworth, A. Badhan, A. A. Boakye et al., "Clinical utility of existing and second-generation interferon- $\gamma$ release assays for diagnostic evaluation of tuberculosis: an observational cohort study," The Lancet Infectious Diseases, vol. 19, no. 2, pp. 193-202, 2019.

[22] M. Zhang, D. Li, Z. D. Hu, and Y. L. Huang, "The diagnostic utility of pleural markers for tuberculosis pleural effusion," Annals of translational medicine., vol. 8, no. 9, p. 607, 2020.

[23] G. J. Fei, L. F. Zhang, and H. J. Shu, "Values of different laboratory diagnostic approaches for tuberculous peritonitis," Zhongguo Yi Xue Ke Xue Yuan Xue Bao, vol. 40, no. 4, pp. 534-538, 2018.

[24] I. Afsar, M. Gunes, H. Er, and A. G. Sener, "Comparison of culture, microscopic smear and molecular methods in diagnosis of tuberculosis," Revista Española de Quimioterapia, vol. 31, no. 5, pp. 435-438, 2018.

[25] M. Pai, C. M. Denkinger, S. V. Kik et al., "Gamma interferon release assays for detection of Mycobacterium tuberculosis infection," Clinical Microbiology Reviews, vol. 27, no. 1, pp. 3-20, 2014.

[26] J. L. De Oliveira, D. A. De Souza Rangel, R. A. Cordeiro et al., "Tuberculous lymphadenopathy mimicking disease activity in a patient with Behçet's disease," Adv Rheumatol., vol. 58, 2018.

[27] İ. Gönen, "Disseminated Tuberculosis During the Course of Behçet's Disease: A Case Report," Turkish Journal of Rheumatology, vol. 27, no. 1, pp. 70-73, 2012.

[28] M. Wang, "A case report of Behcet syndrome with osteoarticular tuberculosis," J New Med., vol. 24, no. 12, pp. 637-638, 1993.

[29] S. Liu, J. Ren, X. Wu et al., "Preliminary Case-control Study to Evaluate Diagnostic Values of C-Reactive Protein and Erythrocyte Sedimentation Rate in Differentiating Active Crohn's Disease From Intestinal Lymphoma, Intestinal Tuberculosis and Behcet's Syndrome," The American Journal of the Medical Sciences, vol. 346, no. 6, pp. 467-472, 2013.

[30] L. L. Zhuang, H. M. Liu, G. M. Li et al., "Dural sinus thrombosis and giant pulmonary artery aneurysm in paediatric Behçet's disease," Clinical and Experimental Rheumatology, vol. 38, no. 3, pp. 558-566, 2020.

[31] Y. Korogi, N. Tanaka, H. Yoshifuji, J. Tazaki, T. Kubo, and K. Tanizawa, "Complete occlusion of right pulmonary artery in Behçet disease," Respirology case reports., vol. 8, no. 6, article e00594, 2020.

[32] A. Iliopoulos, S. Kedikoglou, S. Laxanis, S. Kourouklis, and E. Katsaros, "A case of tuberculous meningoencephalitis in a patient with Behçet's disease," Clinical Rheumatology, vol. 25, no. 1, pp. 121-122, 2006.
[33] S. Wang, J. Wu, J. Chen et al., "Evaluation of Mycobacterium tuberculosis-specific antibody responses for the discrimination of active and latent tuberculosis infection," International Journal of Infectious Diseases, vol. 70, pp. 1-9, 2018.

[34] W. Long, F. Cai, X. Wang, N. Zheng, and R. Wu, "High risk of activation of latent tuberculosis infection in rheumatic disease patients," Infectious Diseases, vol. 52, no. 2, pp. 80-86, 2020.

[35] N. Miyasaka, T. Takeuchi, and K. Eguchi, "Guidelines for the proper use of etanercept in Japan," Modern Rheumatology, vol. 16, no. 2, pp. 63-67, 2014.

[36] J. J. Cush, "Biological drug use: US perspectives on indications and monitoring," Annals of the Rheumatic Diseases, vol. 64, Suppl_4, pp. iv18-iv23, 2005. 